\title{
ASSOCIATION OF MMP-7 -181A> G POLYMORPHISM WITH COLORECTAL CANCER AND GASTRIC CANCER SUSCEPTIBILITY: A SYSTEMATIC REVIEW AND META-ANALYSIS
}

\author{
Associação do polimorfismo MMP-7 -181A> G com câncer colorretal e suscetibilidade ao câncer gástrico: revisão sistemática e \\ metanálise
}

Mohammad ZARE 1 , Jamal JAFARI-NEDOOSHAN ${ }^{1}$, Kazem AGHILI ${ }^{2}$, Hossein AHRAR ${ }^{2}$, Mohammad Hossein JARAHZADEH ${ }^{3}$, Neda SEIFI-SHALAMZARI ${ }^{4}$, Masoud ZARE-SHEHNEH ${ }^{5}$, Hossein NEAMATZADEH ${ }^{5}$

How to cite this article: Zare M, Jafari-Nedooshan, Aghili K, Ahrar H, Jarahzadeh MH, Seifi-Shalamzari N, Zare-Shehneh M, Neamatzadeh H. Association of MMP-7 -181a>G polymorphism with colorectal cancer and gastric cancer susceptibility: a systematic review and meta-analysis. ABCD Arq Bras Cir Dig. 2019;32(3):e1449. DOI: /10.1590/0102-672020190001e1449

From the ${ }^{1}$ Shahid Sadoughi University of Medical Sciences, General Surgery, Yazd, Yazd, Iran; ${ }^{2}$ Shahid Sadoughi University of Medical Sciences, Radiology, Yazd, Yazd, Iran; ${ }^{3}$ Shahid Sadoughi University of Medical Sciences, Anesthesiology and Critical Care, Yazd, Yazd, Iran; ${ }^{4}$ Shahrekord University of Medical Sciences, Emergency Medicine, Yazd, Yazd, Iran, ${ }^{5}$ Sadoughi University of Medical Sciences, Medical Genetics; Yazd, Yazd, Iran.

HEADINGS - Matrix metalloproteinase-7. Colorectal neoplasms. Stomach neoplasms. Polymorphism, Single nucleotide. Metaanalysis.
ABSTRACT - Introduction: The matrix metalloproteinase-7 (MMP-7) gene -181A > G polymorphism has been reported to be associated with colorectal cancer (CRC) and gastric cancer (GC) susceptibility, yet the results of these previous results have been inconsistent or controversial. Aim: To elaborate a meta-analysis to assess the association of $-181 \mathrm{~A}>\mathrm{G}$ polymorphism of MMP-7 with CRC and GC risk. Methods: Published literature evaluating the association from PubMed, Web of Science, Google Scholar and other databases were retrieved up to April 25 , 2018. Pooled odds ratio (OR) and $95 \%$ confidence interval $(\mathrm{Cl})$ were calculated using random- or fixed-effects model. Results: A total of 19 case-control studies, which included eleven studies on CRC $(2,169$ CRC cases and 2,346 controls) and eight studies on GC (1,545 GC cases and 2,366 controls) were identified. There was a significant association between MMP-7 $-181 \mathrm{~A}>\mathrm{G}$ polymorphism and $\mathrm{GC}$ risk under the homozygote model (GG vs. AA: $O R=1.672,95 \%$ $\mathrm{Cl} 1.161-2.409, p=0.006$ ) and the recessive model (GG vs. $G A+A A: O R=1.672,95 \% \mathrm{Cl} 1.319$ $2.554, p=0.001$ ), but not with CRC. By subgroup analysis based on ethnicity, an increased risk of CRC and GC was found only among Asians. Conclusions: This meta-analysis suggests that MMP-7 $-181 \mathrm{~A}>\mathrm{G}$ polymorphisms is associated with GC risk, but not with CRC. However, our results clearly showed that the MMP-7 $-181 \mathrm{~A}>\mathrm{G}$ polymorphism significantly increased the risk of CRC only in Asians.

\section{Correspondência:}

Jamal Jafari-Nedooshan

E-mail:j.jafari.yazd@gmail.com;

fnajafi.ssu@gmail.com

Financial source: none

Conflict of interest none

Received for publication: 11/05/2018

Accepted for publication: 16/01/2019

DESCRITORES - Metaloproteinase $7 \mathrm{da}$ matriz. Neoplasias colorretais. Neoplasias gástricas. Polimorfismo de nucleotídeo único. Metanálise.
RESUMO - Introdução: O polimorfismo da matriz metaloproteinase-7 (MMP-7) -181A>G tem sido relatado como associado à suscetibilidade dos cânceres colorretal (CRC) e gástrico (GC), mas os resultados desses estudos anteriores foram inconsistentes ou controversos. Objetivo: Elaborar metanálise para avaliar a associação do polimorfismo $-181 \mathrm{~A}>\mathrm{G}$ da MMP-7 com o risco de CRC e GC. Métodos: Revisão da literatura publicada avaliando essa associação no PubMed, Web of Science, Google Acadêmico e outras bases de dados até 25 de abril de 2018. Odds ratio (OR) e o intervalo de confiança de $95 \%$ (IC) foram calculados usando dados aleatórios ou modelo de efeitos fixos. Resultados: Um total de 19 estudos caso-controle, que incluíram 11 trabalhos sobre CRC (2.169 casos de CCR e 2.346 controles) e oito sobre GC ( 1.545 casos de GC e 2.366 controles) foram identificados. Houve associação significativa entre o polimorfismo MMP-7 $-181 \mathrm{~A}>\mathrm{G}$ e o risco de GC sob o modelo homozigoto (GG vs. AA: $\mathrm{OR}=1,672$, IC 95\% 1,161-2,409, $p=0,006$ ) e o modelo recessivo (GG vs. GA + AA: OR=1,672, IC $95 \% 1,319-2,554, p=0,001)$, mas não com CRC. Por análise de subgrupos com base na etnia, um risco aumentado de CRC e GC foi encontrado apenas entre os asiáticos. Conclusões: Esta metanálise sugere que os polimorfismos MMP-7 -181A>G estão associados ao risco de GC, mas não ao CRC. No entanto, estes resultados mostraram claramente que o polimorfismo MMP-7 -181A>G aumentou significativamente o risco de CRC apenas em asiáticos.

\section{INTRODUCTION}

$\mathrm{N}$ owadays, gastrointestinal-related cancers especially gastric cancer (GC) and colorectal cancer (CRC) are major causes of cancer-related death worldwide ${ }^{7,22,23}$. Globally, CRC and GC are the third and fourth leading cause most common cancers, making up $10 \%$ and $7 \%$ of cases all new cancer cases, respectively ${ }^{23,27}$. It is well-known that the development of CRC and GC can be induced by the interactions of multiple genetic and environmental factors in complex ways. However, the pathogenesis of CRC differs from that of $G C$ in that it is affected by ethnic background, regional, life style, environmental factors and molecular pathogenesis ${ }^{27}$. Currently, several genes have been reported to be associated with CRC and GC, and the matrix metalloproteinases (MMPs) genes has received increasing attention ${ }^{10,29,31}$.

MMPs are classified as a large family of zinc-containing proteases, which involved in normal physiological and pathological processes such as degradation and 
remolding of extracellular matrix, embryonic development, reproduction and cancer ${ }^{6,13}$. MMP-7, the smallest member of MMP family, is an endopeptidases with broad substrate specificity, which break down extracellular matrix (ECM) by degrading macromolecules including casein, type I, II, IV, and $V$ gelatins, fibronectin, and proteoglycan ${ }^{18}$. In addition, MMP-7 is one of the main regulatory enzymes involved in apoptosis by releases the Fas ligand (FasL) from the membrane then induces apoptosis of neighboring cells, or decreases cancer-cell apoptosis ${ }^{21}$. Thus, MMP-7 promotes cell survival by resisting apoptosis through cleaving FasL. MMP7 is potentially involved in tumor metastasis and inflammatory processes ${ }^{10,31}$.

MMP-7 gene (also known as matrilysin) is localized on chromosome 11q21-q22 and contains 13 exons $^{35}$. The single nucleotide polymorphism (SNP) $-181 \mathrm{~A}>\mathrm{G}$ in promoter region of MMP-7 gene has been considered to be a candidate SNP for various conditions including gastrointestinal related malignancies ${ }^{1,10,31}$. There is clear evidence that MMP-7 gene up-regulation is significantly related to the promoter activity variation of the $-181 \mathrm{~A}>\mathrm{G} 16$. Molecular epidemiological studies have reported the association of MMP-7 -181A>G polymorphism with $C R C$ and $G C$ risk, but the results remain conflicting rather than conclusive. Several studies previously have performed on the association of MMP-7 -181A>G polymorphism with $C R C$ and GC risk ${ }^{10,31}$. However, these studies had opposite observations and additional casecontrol studies with larger sample sizes have been published since then. Hence, the association of MMP-7 -181A>G polymorphism remains unknown.

Therefore, we have performed a meta-analysis of all eligible studies to derive more precise estimation of the association of MMP-7 -181A $>\mathrm{G}$ polymorphism with CRC and GC risk.

METHODS

\section{Literature search}

Following PRISMA guidance, we searched the electronic literature databases including PubMed, EMBASE, Elsevier, Science Direct, Wan Fang, Chinese National Knowledge Infrastructure (CNKI) and Chinese Biomedical Literature for all relevant articles published up to April 25, 2018. The search strategies were based on combinations of the following key words: ("matrix metalloproteinase-7" OR "MMP-7" OR "matrilysin" OR uterine metalloproteinase OR "pump-1 protease" OR "PUMP-1") AND ("-181A>G OR "rs11568818") AND ("colorectal cancer" OR "CRC") AND ("gastric adenocarcinoma" OR "stomach cancer" OR" gastric cancer" OR "GC") AND ("gene" or "allele" or "genotype" or "mutation" or "variant" or "variation" or "polymorphism"), without any restriction on language. Review articles were hand-searched to find additional eligible studies and only published studies with full-text articles were included. We excluded studies that were not full-length publications articles or letters in peer-reviewed English journals. When the same patient population was included in different articles, the one with the largest population of participants or the most recent one was selected.

\section{Inclusion and exclusion criteria}

Inclusion criteria was defined as follows: 1) published studies and contained original data; 2 ) case-control studies; 3 ) evaluating the association of MMP-7 -181A $>\mathrm{G}$ polymorphism with $C R C$ and GC risk; and 4) sufficient published data available to estimate an odds ratio (OR) with $95 \%$ confidence interval $(\mathrm{Cl})$. Major reasons for exclusion of studies were as follows: 1) only case population, family based or linkage studies; 2 ) studies that could not offer the number of cases and controls or other essential data; 3 ) reviews, abstracts, letters to editor, case reports or animal studies; 4 ) duplicate of previous publication or studies with overlapping patient populations; and 5) studies without histologically confirmed of CRC and GC. For more than two studies with overlapping data, the study with the most subjects or newest published data was selected.

\section{Data extraction}

Data were carefully extracted from all eligible publications by two of the authors independently. If the study provided stratum information, the data coming from similar stratum were added up to make full use of the data. Disagreements between the two authors were resolved by discussing the results with a third one. For each study, the following variables were collected: first author's name, year of publication, country, ethnicity of participants, number of cases and controls, genotyping methods, and allele numbers and genotype distributions in cases and controls, minor allele frequencies (MAFs) in control subjects, and the results of Hardy-Weinberg equilibrium (HWE) test. Different ethnicities were categorized as Asian, Caucasian and Latinos (mixed). Study designs were stratified to population-based studies and hospital-based studies.

\section{Statistical analysis}

The strength of the association of MMP-7 -181A>G polymorphism with $C R C$ and $G C$ risk was measured using odds ratios (ORs) with $95 \%$ confidence intervals (Cls). The statistical significance of the pooled OR was assessed with the Z-test and $p<0.05$ was considered significant. The pooled ORs were performed under five genetic models, i.e., allele ( $G$ vs. A), heterozygote (GA vs. AA), homozygote (GG vs. $A A)$, dominant ( $G G+G A$ vs. $A A$ ) and recessive ( $G G$ vs. $G A+A A$ ). The between-study heterogeneity was evaluated by a chi-square-based $Q$ test, which $p$ value for the $Q$-test less than 0.10 indicates existing heterogeneity among studies. In addition, the $\mathrm{I}^{2}$ statistics was used to quantify the proportion of the total variation across studies due to heterogeneity. A high value of 12 indicated a higher probability of the existence of heterogeneity $\left(\mathrm{I}^{2}=0 \%\right.$ to $25 \%$, no heterogeneity; $\mathrm{I}^{2}=25 \%$ to $50 \%$, moderate heterogeneity; $\mathrm{I}^{2}=50 \%$ to $75 \%$, large heterogeneity; and $\mathrm{I}^{2}=75 \%$ to $100 \%$, extreme heterogeneity). A random-effects (DerSimonianLaird method) or fixed-effects (Mantel-Haenszel method) model was used to calculate pooled effect estimates in the presence or absence of heterogeneity. HWE of genotype distribution in the controls of included studies was conducted using by Pearson's $x^{2}$ test, in which $p$-value less than 0.05 was considered significantly deviating from HWE. Subgroup analyses were performed by ethnicity, genotyping method, HWE status, source of controls and cancer type (CRC and GC). In addition, to consider the possible sources of heterogeneity, the studies we stratified. To validate the reliability of the results, sensitivity analysis was performed though omitting one case-control study each time, as well as limiting this meta-analysis to studies which were conformed to HWE. Funnel plots and Egger's linear regression test were used to diagnose potential publication bias $(p<0.05)$. All analyses were performed with the comprehensive meta-analysis (CMA) 2.0 software (Biostat, USA). Two-sided $p$-values $<0.05$ were considered statistically significant.

\section{RESULTS}

\section{Extraction process and study characteristics}

The flow diagram of literature search was given in Figure 1. The initial search of databases yielded 103 relevant publications based on our literature search strategy, and an 
additional one study was identified through hand searching. However, 36 of them were ruled out because of duplicate results obtained from multiple databases, 68 articles remained. In addition, after the titles and abstracts of the 68 articles were reviewed, 49 full-text irrelevant studies were excluded. Finally, 19 eligible case-control studies with 3,714 cases and 4,712 controls were included in this meta-analysis. The characteristics of studies included in the current metaanalysis are shown in Table 1. Among these studies, eleven studies with 2,169 CRC cases and 2,346 controls were on $\mathrm{CRC}^{2-4,8,16,19,20,24,28,33}$, and eight studies with 1,545 GC cases and 2,366 controls were on $\mathrm{GC}^{5,11,12,14,15,17,26,34}$. By ethnics, there were 13 studies of Asians (countries: Korea, Japan, China, Iran, Kashmir, Taiwan, and India), four studies of Caucasians (countries: Italy, France, Poland and Netherland), and two studies of Latinos (countries: Brazil and Mexico). According to the control source, ten studies were hospital-based, eight studies were population-based and one study was not clear. The studies used four different genotyping methods including direct sequencing, TaqMan, polymerase chain reaction-restriction fragment length polymorphism (PCR-RFLP) analysis and tetra-primer amplification refractory mutation system-polymerase chain (ARMS-PCR). All of the studies indicated that the distribution of genotypes in the controls was consistent with HWE except for two studies (Table 1).

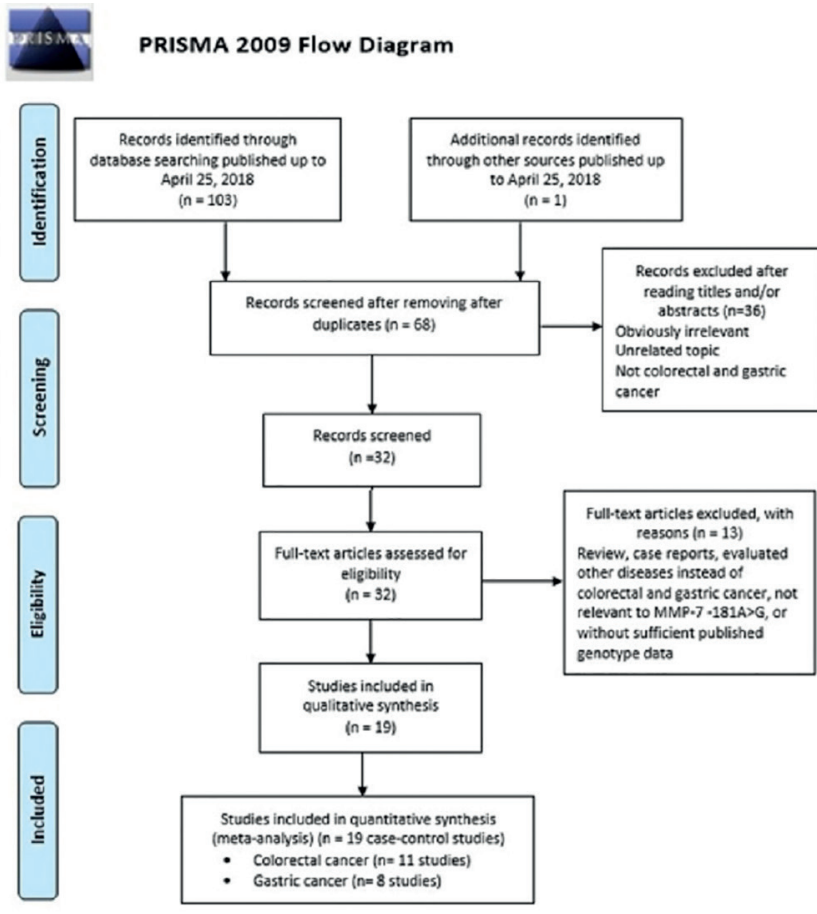

FIGURE 1 - The study selection and inclusion process.

Quantitative synthesis

Overall study

Table 2 listed the main results of the meta-analysis of MMP-7 -181A $>$ G polymorphism with CRC and GC risk. We pooled all the 19 case-control studies together to assess the overall association of MMP-7 $-181 \mathrm{~A}>\mathrm{G}$ polymorphism with $C R C$ and GC risk. Overall, no significant main effects on CRC and GC susceptibility were observed in the overall population under all the five genetic models, i.e., allele $(G$ vs. $A: O R=1.049,95 \% \mathrm{Cl} 0.889-1.239, p=0.570$, Figure $2 A$ ), heterozygote (GA vs. AA: OR=1.083, $95 \% \mathrm{Cl} 0.813-1.443$, $\mathrm{p}=0.586)$, homozygote (GG vs. $A A: O R=0.982,95 \% \mathrm{Cl} 0.701$ $1.375, p=0.915)$, dominant (GG+GA vs. $A A: O R=1.061,95 \% \mathrm{Cl}$ $0.869-1.296, p=559)$ and recessive (GG vs. $G A+A A: O R=1.084$, $95 \% \mathrm{Cl} 0.786-1.495, \mathrm{p}=0.622)$.
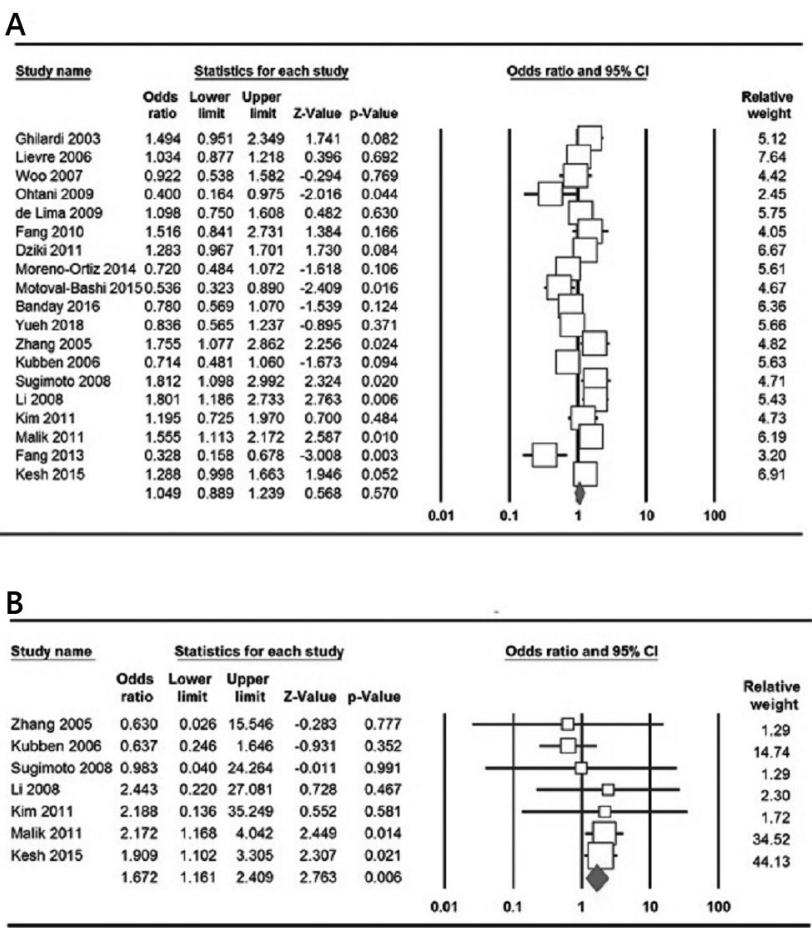

FIGURE 2 - Forest plot for the association of MMP-7 -181A>G polymorphism with $C R C$ and $G C$ risk: $A$ ) the allele model ( $G$ vs. A) in overall estimations; $B$ ) the homozygote model (GG vs. AA) in GC studies

\section{Colorectal cancer}

Table 2 also listed the main results of the meta-analysis of MMP-7 -181A > G polymorphism with CRC risk. When all the 11 eligible studies were pooled into the meta-analysis of MMP-7 -181A $>G$ polymorphism, we have not found evidence of a significant MMP-7 $-181 \mathrm{~A}>\mathrm{G}$ polymorphism with CRC risk under all the five genetic models. In the subgroup analysis by ethnicity, significantly increased risk of CRC was observed in Asians under three genetic models, i.e., allele $(G$ vs. $A: O R=0.798,95 \% \mathrm{Cl} 0.661-0.964, p=0.019$, Figure $2 A)$, homozygote (GG vs. AA: OR=0.490, 95\% Cl 00.286-0.838, $\mathrm{p}=0.009$ ) and recessive model (GG vs. $G A+A A: O R=0.530$, $95 \% \mathrm{Cl}$ 0.340-0.826, $\mathrm{p}=0.005)$, but not in Caucasians and Latinos populations (Table 2).

We also performed subgroup analyses based on the source of control and genotyping method, when it was available (Table 3). The hospital-based subgroup analysis revealed that the presence of the MMP-7 -181A $>$ G polymorphism was related to a higher risk of CRC under the homozygote model (GG vs. AA: OR=0.671, 95\% Cl 0.484-0.951, $\mathrm{p}=0.023$ ). In the PCR-RFLP group, significantly increased association between MMP-7 -181A $>G$ polymorphism and $C R C$ risk was found under the homozygote model (GG vs. AA: OR=0.680, 95\% Cl 0.486-0.950, $\mathrm{p}=0.024$, Table 3).

\section{Gastric cancer}

Table 2 also listed the main results of the meta-analysis of MMP-7 -181A $>\mathrm{G}$ polymorphism with $\mathrm{GC}$ risk. There was a significant association between MMP-7 -181A>G polymorphism and $\mathrm{GC}$ risk under two genetic models, i.e., homozygote (GG vs. AA: OR=1.672, 95\% Cl 1.161-2.409, $p=0.006$, Fig $2 B$ ) and recessive ( $G G$ vs. $G A+A A: O R=1.835$, $95 \% \mathrm{Cl} 1.319-2.554, p=0.001)$. Similarly, when stratified by ethnicity, a significant association between MMP-7 -181A>G polymorphism and increased risk of GC was detected among Asians under the homozygote model (GG vs. AA: OR $=1.975$, 95\% $\mathrm{Cl} 1.331-2.934, \mathrm{p}=0.006)$ and the recessive model (GG vs. $G A+A A: O R=2.022,95 \% \mathrm{Cl} 1.416-2.886, p=0.001)$.

The studies were further stratified on the basis of 
TABLE 1 - Main characteristics of all studies included in the meta-analysis

\begin{tabular}{|c|c|c|c|c|c|c|c|c|c|c|c|c|c|c|c|c|}
\hline \multirow{3}{*}{ First Author } & \multirow{3}{*}{$\begin{array}{l}\text { Country } \\
\text { (Ethnicity) }\end{array}$} & \multirow{3}{*}{$\begin{array}{l}\text { Genotyping } \\
\text { Technique }\end{array}$} & \multirow{3}{*}{ SOC } & \multirow{3}{*}{$\begin{array}{l}\text { Case/ } \\
\text { Control }\end{array}$} & \multicolumn{5}{|c|}{ Cases } & \multicolumn{5}{|c|}{ Controls } & \multirow{3}{*}{ MAFs } & \multirow{3}{*}{ HWE } \\
\hline & & & & & \multicolumn{3}{|c|}{ Genotype } & \multicolumn{2}{|c|}{ Allele } & \multicolumn{3}{|c|}{ Genotype } & \multicolumn{2}{|c|}{ Allele } & & \\
\hline & & & & & AA & AG & GG & A & G & AA & $A G$ & GG & A & G & & \\
\hline \multicolumn{17}{|l|}{$\begin{array}{l}\text { Colorectal } \\
\text { Cancer }\end{array}$} \\
\hline Ghilardi 2003 & $\begin{array}{c}\text { Italy } \\
\text { (Caucasian) }\end{array}$ & Sequencing & PB & $58 / 111$ & 15 & 28 & 15 & 58 & 58 & 36 & 61 & 14 & 133 & 89 & 0.400 & 0.129 \\
\hline Lievre 2006 & $\begin{array}{c}\text { France } \\
\text { (Caucasian) }\end{array}$ & TaqMan & PB & $596 / 565$ & 191 & 272 & 131 & 658 & 534 & 187 & 259 & 119 & 1083 & 497 & 0.439 & 0.097 \\
\hline Woo 2007 & $\begin{array}{l}\text { Korea } \\
\text { (Asian) }\end{array}$ & PCR-RFLP & PB & $185 / 304$ & 163 & 22 & 0 & 348 & 22 & 265 & 39 & 0 & 565 & 39 & 0.064 & 0.232 \\
\hline Ohtani 2009 & $\begin{array}{l}\text { Japan } \\
\text { (Asian) }\end{array}$ & PCR-RFLP & $\mathrm{HB}$ & $119 / 67$ & 110 & 9 & 0 & 229 & 9 & 55 & 12 & 0 & 122 & 12 & 0.089 & 0.420 \\
\hline de Lima 2009 & $\begin{array}{c}\text { Brazil } \\
\text { (Latinos) }\end{array}$ & PCR-RFLP & HB & $108 / 113$ & 36 & 56 & 16 & 128 & 88 & 41 & 57 & 15 & 139 & 87 & 0.384 & 0.487 \\
\hline Fang 2010 & $\begin{array}{l}\text { China } \\
\text { (Asian) }\end{array}$ & PCR-RFLP & PB & $252 / 237$ & 22 & 30 & 0 & 474 & 30 & 218 & 19 & 0 & 455 & 19 & 0.040 & 0.520 \\
\hline Dziki 2011 & $\begin{array}{l}\text { Poland } \\
\text { (Caucasian) }\end{array}$ & PCR-RFLP & HB & $184 / 205$ & 99 & 93 & 52 & 171 & 197 & 66 & 94 & 45 & 216 & 194 & 0.473 & 0.294 \\
\hline $\begin{array}{l}\text { Moreno-Ortiz } \\
\quad 2014\end{array}$ & $\begin{array}{l}\text { México } \\
\text { (Latinos) }\end{array}$ & PCR-RFLP & HB & $102 / 121$ & 46 & 51 & 5 & 143 & 61 & 49 & 54 & 18 & 152 & 90 & 0.371 & 0.622 \\
\hline $\begin{array}{l}\text { Motoval-Bashi } \\
2015\end{array}$ & $\begin{array}{c}\text { Iran } \\
\text { (Asian) }\end{array}$ & ARMS-PCR & NS & $61 / 77$ & 11 & 31 & 19 & 53 & 69 & 6 & 40 & 31 & 52 & 102 & 0.708 & 0.156 \\
\hline Banday 2016 & $\begin{array}{l}\text { Kashmir } \\
\text { (Asian) }\end{array}$ & PCR-RFLP & $\mathrm{HB}$ & $142 / 184$ & 43 & 82 & 13 & 176 & 108 & 61 & 84 & 39 & 206 & 162 & 0.440 & 0.317 \\
\hline Yueh 2018 & $\begin{array}{l}\text { Taiwan } \\
\text { (Asian) }\end{array}$ & PCR-RFLP & $\mathrm{HB}$ & $362 / 362$ & 318 & 38 & 6 & 674 & 50 & 311 & 43 & 8 & 665 & 59 & 0.081 & $=0.001$ \\
\hline \multicolumn{17}{|l|}{ Gastric Cancer } \\
\hline Zhang 2005 & $\begin{array}{l}\text { China } \\
\text { (Asian) }\end{array}$ & PCR-RFLP & PB & $201 / 350$ & 167 & 34 & 0 & 368 & 34 & 316 & 33 & 1 & 662 & 35 & 0.050 & 0.888 \\
\hline Kubben 2006 & $\begin{array}{l}\text { Nederland } \\
\text { (Caucasian) }\end{array}$ & PCR-RFLP & PB & $79 / 169$ & 34 & 37 & 8 & 105 & 53 & 46 & 106 & 17 & 198 & 140 & 0.414 & $=0.001$ \\
\hline Sugimoto 2008 & $\begin{array}{l}\text { Japan } \\
\text { (Asian) }\end{array}$ & PCR-RFLP & HB & $160 / 434$ & 133 & 27 & 0 & 293 & 27 & 393 & 40 & 1 & 826 & 42 & 0.048 & 0.986 \\
\hline Li 2008 & $\begin{array}{l}\text { China } \\
\text { (Asian) }\end{array}$ & PCR-RFLP & PB & $338 / 380$ & 280 & 56 & 2 & 616 & 60 & 342 & 37 & 1 & 721 & 39 & 0.051 & 0.999 \\
\hline Kim 2011 & $\begin{array}{l}\text { Korea } \\
\text { (Asian) }\end{array}$ & PCR-RFLP & HB & $153 / 326$ & 128 & 24 & 1 & 280 & 26 & 280 & 45 & 1 & 605 & 47 & 0.072 & 0.565 \\
\hline Malik 2011 & $\begin{array}{l}\text { India } \\
\text { (Asian) }\end{array}$ & PCR-RFLP & PB & $108 / 195$ & 29 & 39 & 40 & 98 & 119 & 63 & 92 & 40 & 218 & 172 & 0.441 & 0.547 \\
\hline Fang 2013 & $\begin{array}{l}\text { China } \\
\text { (Asian) }\end{array}$ & PCR-RFLP & $\mathrm{HB}$ & $246 / 252$ & 236 & 10 & 0 & 482 & 10 & 222 & 30 & 0 & 474 & 30 & 0.059 & 0.315 \\
\hline Kesh 2015 & $\begin{array}{l}\text { India } \\
\text { (Asian) }\end{array}$ & PCR-RFLP & HB & $260 / 260$ & 107 & 108 & 45 & 322 & 198 & 118 & 116 & 26 & 352 & 168 & 0.323 & 0.746 \\
\hline
\end{tabular}

PCR-RFLP=polymerase chain reaction-restriction fragment length polymorphism; ARMS-PCR=tetra-primer amplification refractory mutation system-polymerase chain; $\mathrm{SOC}=$ source of control; $\mathrm{HB}=$ hospital-based; $\mathrm{PB}=$ population-based; $\mathrm{MAF}=$ minor allele frequency; $\mathrm{HWE}=$ Hardy-Weinberg equilibrium; $\mathrm{NS}=$ not stated

source of controls (Table 3). When stratifying by source of control, a significant association between MMP-7 -181A>G polymorphism and increased risk of $\mathrm{GC}$ was detected in population-based studies under the recessive model (GG vs. $G A+A A: O R=1.819,95 \% \mathrm{Cl} 1.173-2.819, p=0.008)$, and in hospital-based studies under two genetic models, i.e., homozygote (GG vs. AA: OR=1.884, 95\% Cl 1.107-3.204, $p=0.020)$ and recessive ( $G G$ vs. $G A+A A: O R=1.858,95 \% \mathrm{Cl}$ 1.124-3.069, $p=0.016)$.

\section{Heterogeneity analysis}

Heterogeneity was detected among studies under all the five genetic models, i.e., allele (G vs. A: $12=70.90 \%$, $\mathrm{PH}=0.001)$, heterozygote (GA vs. $\mathrm{AA}: \mathrm{I} 2=82.36 \%, \mathrm{PH}=0.001)$, homozygote (GG vs. $A A: 12=54.65 \%, P H=0.006$ ), dominant ( $G G+G A$ vs. $A A: 12=65.43 \%, P H=0.001)$ and recessive $(G G$ vs. $\mathrm{GA}+\mathrm{AA}: \mathrm{I2}=65.4 \%, \mathrm{PH}=0.001)$. Thus, to explore the potential sources of heterogeneity across studies, we assessed the pooled ORs via stratification by cancer type, ethnicity, genotyping method, HWE status and source of controls. The results showed that the heterogeneity effectively removed by subgroup analyses based on ethnicity among studies on CRC. Therefore, we found that genotyping method, HWE status and source of controls did not contribute to substantial heterogeneity among the meta-analysis.

\section{Sensitivity analysis}

To evaluate the effect of individual study on the pooled ORs and stability of the meta-analysis results, we excluded one study at a time. However, the omission of any single study made no significant difference, suggesting that the results of this meta-analysis were stable. Moreover, sensitivity analysis was performed after excluding HWE-violating studies, and the corresponding pooled ORs were not qualitatively altered (data not shown).

\section{Publication bias}

Both Begg's funnel plot and Egger's test were performed to assess the publication bias of literature. Begg's funnel plots did not reveal any evidence of obvious asymmetry under all five genetic models in the overall meta-analysis. For example, the shape of the funnel plot did not indicate any evidence of obvious asymmetry under the allele model (Figure 3), and the Egger's test suggested the absence of publication bias (PBeggs $=0.293$ and PEggers $=0.483$ ). However, the results of Egger's regression test showed evidence of publication bias among Caucasian's studies on CRC under the recessive model (GG vs. GA+AA: PBegg's=0.296, PEggers=0.001). 
TABLE 2 - Meta-analysis for the association of MMP-7 -181A>G polymorphism with CRC and GC risk

\begin{tabular}{|c|c|c|c|c|c|c|c|c|c|c|}
\hline \multirow{2}{*}{ Subgroup } & \multirow{2}{*}{ Genetic model } & \multirow{2}{*}{$\begin{array}{l}\text { Type of } \\
\text { model }\end{array}$} & \multicolumn{2}{|c|}{ Heterogeneity } & \multicolumn{4}{|c|}{ Odds ratio (OR) } & \multicolumn{2}{|c|}{ Publication bias } \\
\hline & & & $12(\%)$ & $\mathrm{PH}$ & OR & $95 \% \mathrm{Cl}$ & ZOR & POR & PBeggs & PEggers \\
\hline \multirow[t]{5}{*}{ Overall $(n=19)$} & G vs. A & Random & 70.90 & $=0.001$ & 1.049 & $0.889-1.239$ & 0.568 & 0.570 & 0.293 & 0.483 \\
\hline & GA vs. AA & Random & 82.36 & $=0.001$ & 1.083 & $0.813-1.443$ & 0.545 & 0.586 & 0.293 & 0.906 \\
\hline & GG vs. AA & Random & 54.65 & 0.006 & 0.982 & $0.701-1.375$ & -0.107 & 0.915 & 0.766 & 0.611 \\
\hline & $G G+G A$ vs. $A A$ & Random & 65.43 & $=0.001$ & 1.061 & $0.869-1.296$ & 0.585 & 0.559 & 0.068 & 0.223 \\
\hline & GG vs. $G A+A A$ & Random & 60.48 & 0.001 & 1.084 & $0.786-1.495$ & 0.494 & 0.622 & 0.692 & 0.651 \\
\hline \multirow[t]{5}{*}{$\begin{array}{c}\text { Colorectal } \\
\text { cancer }(n=11)\end{array}$} & G vs. A & Random & 58.32 & 0.008 & 0.947 & $0.791-1.133$ & -0.593 & 0.553 & 0.275 & 0.345 \\
\hline & GA vs. AA & Random & 85.27 & $=0.001$ & 1.101 & $0.721-1.682$ & 0.444 & 0.657 & 0.876 & 0.779 \\
\hline & GG vs. AA & Random & 58.51 & 0.018 & 0.791 & $0.529-1.183$ & -1.142 & 0.253 & 0.536 & 0.330 \\
\hline & $G G+G A$ vs. $A A$ & Fixed & 42.24 & 0.068 & 1.032 & $0.894-1.192$ & 0.431 & 0.667 & 0.533 & 0.355 \\
\hline & $G G$ vs. $G A+A A$ & Random & 68.09 & 0.003 & 0.878 & $0.589-1.309$ & -0.639 & 0.523 & 0.901 & 0.401 \\
\hline \multirow[t]{5}{*}{$\begin{array}{l}\text { Gastric cancer } \\
\qquad(n=8)\end{array}$} & G vs. A & Random & 76.10 & $=0.001$ & 1.215 & $0.897-1.645$ & 1.256 & 0.209 & 0.901 & 0.459 \\
\hline & GA vs. $A A$ & Random & 79.18 & $=0.001$ & 1.063 & $0.711-1.590$ & 0.300 & 0.764 & 0.035 & 0.233 \\
\hline & GG vs. AA & Fixed & 0.00 & 0.485 & 1.672 & $1.161-2.409$ & 2.763 & 0.006 & 0.229 & 0.499 \\
\hline & $G G+G A$ vs. $A A$ & Random & 78.48 & $=0.001$ & 1.132 & $0.771-1.660$ & 0.632 & 0.527 & 0.107 & 0.222 \\
\hline & GG vs. $G A+A A$ & Fixed & 0.00 & 0.789 & 1.835 & $1.319-2.554$ & 3.604 & $=0.001$ & 0.367 & 0.310 \\
\hline \multicolumn{11}{|l|}{ Colorectal Cancer } \\
\hline \multirow[t]{5}{*}{ Asian $(n=6)$} & G vs. A & Fixed & 47.83 & 0.088 & 0.798 & $0.661-0.964$ & -2.337 & 0.019 & 0.707 & 0.796 \\
\hline & GA vs. AA & Random & 91.80 & $=0.001$ & 1.216 & $0.486-3.040$ & 0.416 & 0.676 & 0.707 & 0.959 \\
\hline & GG vs. AA & Fixed & 0.00 & 0.612 & 0.490 & $0.286-0.838$ & -2.606 & 0.009 & 1.000 & 0.995 \\
\hline & $G G+G A$ vs. $A A$ & Fixed & 46.52 & 0.096 & 0.896 & $0.708-1.134$ & -0.915 & 0.360 & 0.707 & 0.189 \\
\hline & GG vs. $G A+A A$ & Fixed & 0.00 & 0.397 & 0.530 & $0.340-0.826$ & -2.808 & 0.005 & 1.000 & 0.587 \\
\hline \multirow[t]{5}{*}{ Caucasian $(n=3)$} & G vs. A & Fixed & 40.68 & 0.185 & 1.123 & $0.981-1.285$ & 1.676 & 0.094 & 0.296 & 0.126 \\
\hline & GA vs. AA & Fixed & 38.97 & 0.194 & 0.923 & $0.744-1.143$ & -0.736 & 0.461 & 1.000 & 0.844 \\
\hline & GG vs. AA & Fixed & 59.28 & 0.086 & 1.054 & $0.812-1.366$ & 0.393 & 0.694 & 1.000 & 0.643 \\
\hline & $G G+G A$ vs. $A A$ & Fixed & 53.13 & 0.118 & 1.180 & $0.960-1.451$ & 1.569 & 0.117 & 1.000 & 0.460 \\
\hline & $G G$ vs. $G A+A A$ & Fixed & 51.03 & 0.130 & 1.210 & $0.962-1.522$ & 1.628 & 0.104 & 0.296 & 0.001 \\
\hline \multirow[t]{5}{*}{ Latinos $(n=2)$} & G vs. A & Fixed & 55.62 & 0.133 & 0.897 & $0.681-1.181$ & -0.773 & 0.440 & NA & NA \\
\hline & GA vs. AA & Fixed & 0.00 & 0.795 & 1.059 & $0.709-1.581$ & 0.278 & 0.781 & NA & NA \\
\hline & GG vs. AA & Random & 75.98 & 0.041 & 0.625 & $0.157-2.488$ & -0.667 & 0.505 & NA & NA \\
\hline & $\mathrm{GG}+\mathrm{GA}$ vs. $A \mathrm{~A}$ & Fixed & 0.00 & 0.476 & 0.947 & $0.645-1.392$ & -0.276 & 0.783 & NA & NA \\
\hline & GG vs. $G A+A A$ & Random & 76.58 & 0.039 & 0.606 & $0.162-2.267$ & -0.743 & 0.457 & NA & NA \\
\hline \multicolumn{11}{|l|}{ Gastric Cancer } \\
\hline \multirow[t]{5}{*}{ Asian $(n=7)$} & G vs. A & Random & 69.58 & 0.003 & 1.331 & $0.994-1.782$ & 1.918 & 0.055 & 1.000 & 0.451 \\
\hline & GA vs. AA & Random & 74.59 & 0.001 & 1.197 & $0.815-1.758$ & 0.917 & 0.359 & 0.229 & 0.368 \\
\hline & GG vs. AA & Fixed & 0.00 & 0.976 & 1.976 & $1.331-2.934$ & 3.380 & 0.001 & 0.259 & 0.298 \\
\hline & $G G+G A$ vs. $A A$ & Random & 71.98 & 0.002 & 1.281 & $0.898-1.826$ & 1.367 & 0.172 & 0.367 & 0.341 \\
\hline & GG vs. $G A+A A$ & Fixed & 0.00 & 0.953 & 2.022 & $1.416-2.886$ & 3.877 & $=0.001$ & 0.259 & 0.240 \\
\hline
\end{tabular}

NA=not applicable

TABLE 3 - Meta-analysis for the association of MMP-7 -181A>G polymorphism with CRC and GC risk

\begin{tabular}{|c|c|c|c|c|c|c|c|c|c|c|}
\hline \multirow{2}{*}{ Subgroup } & \multirow{2}{*}{ Genetic model } & \multirow{2}{*}{$\begin{array}{l}\text { Type of } \\
\text { model }\end{array}$} & \multicolumn{2}{|c|}{ Heterogeneity } & \multicolumn{4}{|c|}{ Odds ratio (OR) } & \multicolumn{2}{|c|}{ Publication bias } \\
\hline & & & $12(\%)$ & PH & OR & $95 \% \mathrm{Cl}$ & ZOR & POR & PBeggs & PEggers \\
\hline \multicolumn{11}{|l|}{ Colorectal Cancer } \\
\hline \multirow[t]{5}{*}{ PB $(n=4)$} & G vs. A & Fixed & 21.99 & 0.279 & 1.089 & $0.943-1.257$ & 1.159 & 0.246 & 0.734 & 0.390 \\
\hline & GA vs. AA & Random & 94.02 & $=0.001$ & 1.961 & $0.660-5.829$ & 1.211 & 0.226 & 0.308 & 0.456 \\
\hline & GG vs. AA & Fixed & 65.72 & 0.088 & 1.178 & $0.871-1.595$ & 1.063 & 0.288 & NA & NA \\
\hline & $G G+G A$ vs. $A A$ & Fixed & 0.00 & 0.518 & 1.089 & $0.890-1.333$ & 0.829 & 0.407 & 0.308 & 0.414 \\
\hline & $G G$ vs. $G A+A A$ & Fixed & 71.99 & 0.059 & 1.153 & $0.885-1.503$ & 1.054 & 0.292 & NA & NA \\
\hline \multirow[t]{5}{*}{$H B(n=6)$} & G vs. A & Random & 59.11 & 0.032 & 0.884 & $0.688-1.135$ & -0.969 & 0.333 & 0.060 & 0.118 \\
\hline & GA vs. $A A$ & Fixed & 45.41 & 0.103 & 0.892 & $0.720-1.106$ & -1.039 & 0.299 & 1.000 & 0.585 \\
\hline & GG vs. AA & Fixed & 25.38 & 0.250 & 0.671 & $0.484-0.951$ & -2.260 & 0.023 & 0.806 & 0.583 \\
\hline & $G G+G A$ vs. $A A$ & Random & 56.09 & 0.044 & 0.971 & $0.701-1.347$ & -0.174 & 0.862 & 0.707 & 0.192 \\
\hline & GG vs. $G A+A A$ & Random & 73.18 & 0.005 & 0.702 & $0.367-1.341$ & -1.072 & 0.284 & 0.806 & 0.258 \\
\hline \multirow[t]{5}{*}{ PCR-RFLP $(n=8)$} & G vs. A & Random & 52.42 & 0.040 & 0.934 & $0.751-1.160$ & -0.620 & 0.536 & 0.386 & 0.396 \\
\hline & GA vs. AA & Random & 89.23 & $=0.001$ & 1.212 & $0.665-2.210$ & 0.627 & 0.531 & 0.386 & 0.423 \\
\hline & GG vs. AA & Fixed & 25.41 & 0.252 & 0.680 & $0.486-0.950$ & -2.261 & 0.024 & 0.806 & 0.583 \\
\hline & $G G+G A$ vs. $A A$ & Fixed & 47.45 & 0.065 & 1.022 & $0.785-1.331$ & 0.165 & 0.869 & 0.901 & 0.260 \\
\hline & GG vs. $G A+A A$ & Random & 73.18 & 0.005 & 0.702 & $0.367-1.341$ & -1.072 & 0.284 & 0.806 & 0.258 \\
\hline \multicolumn{11}{|l|}{ Gastric Cancer } \\
\hline \multirow[t]{5}{*}{ PB $(n=4)$} & G vs. A & Random & 77.98 & 0.003 & 1.360 & $0.884-2.093$ & 1.401 & 0.161 & 0.734 & 0.873 \\
\hline & GA vs. AA & Random & 83.08 & $=0.001$ & 1.138 & $0.601-2.155$ & 0.397 & 0.692 & 0.089 & 0.192 \\
\hline & GG vs. AA & Fixed & 39.16 & 0.177 & 1.504 & $0.911-2.484$ & 1.595 & 0.111 & 0.734 & 0.678 \\
\hline & $G G+G A$ vs. $A A$ & Random & 81.36 & 0.001 & 1.244 & $0.693-2.233$ & 0.733 & 0.464 & 0.089 & 0.239 \\
\hline & $G G$ vs. $G A+A A$ & Fixed & 0.00 & 0.400 & 1.819 & $1.173-2.819$ & 2.673 & 0.008 & 0.734 & 0.490 \\
\hline \multirow[t]{5}{*}{$\mathrm{HB}(n=4)$} & G vs. A & Random & 80.17 & 0.002 & 1.048 & $0.622-1.766$ & 0.178 & 0.859 & 0.734 & 0.478 \\
\hline & GA vs. $A A$ & Random & 80.40 & 0.002 & 0.985 & $0.545-1.781$ & -0.049 & 0.961 & 0.734 & 0.576 \\
\hline & GG vs. AA & Fixed & 0.00 & 0.918 & 1.884 & $1.107-3.204$ & 2.336 & 0.020 & 0.296 & 0.644 \\
\hline & $G G+G A$ vs. $A A$ & Random & 81.01 & 0.001 & 1.016 & $0.564-1.831$ & 0.054 & 0.957 & 0.308 & 0.475 \\
\hline & $G G$ vs. $G A+A A$ & Fixed & 0.00 & 0.901 & 1.858 & $1.124-3.069$ & 2.417 & 0.016 & 0.296 & 0.621 \\
\hline
\end{tabular}

$\mathrm{PB}=$ population-based; $\mathrm{HB}=$ hospital-based; $\mathrm{PCR}-\mathrm{RFLP}=$ polymerase chain reaction-restriction fragment length polymorphism; $\mathrm{NA}=$ not applicable 


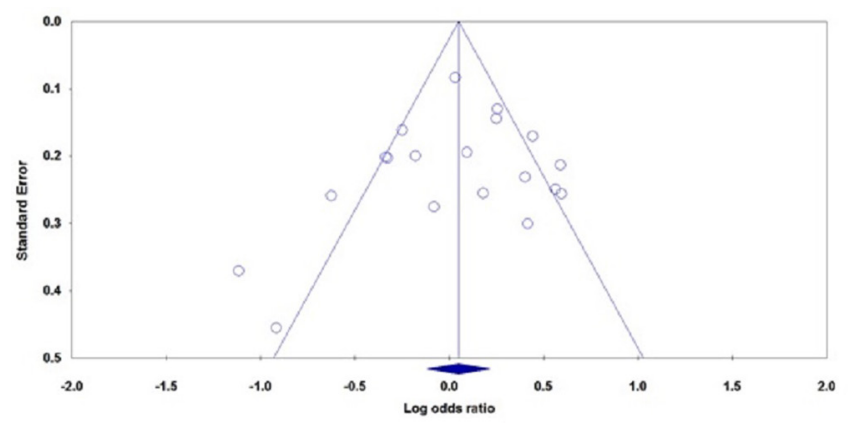

FIGURE 3 - Begg's funnel plots of the MMP-7 -181A>G polymorphism with $C R C$ and $G C$ risk for publication bias test under the allele model (G vs. A): each point represents a separate study for the indicated association

\section{Minor allele frequencies (MAFs)}

The minor allele frequencies (MAFs) of the MMP-7-181A>G polymorphism are shown in Table 2 . The allele and genotype distributions of MMP-7 $-181 \mathrm{~A}>\mathrm{G}$ polymorphism exhibited ethnic variations. The MMP-7 -181A $>$ G polymorphism MAF in overall populations, Asians, Caucasians and Latinos were $37.4 \%$ (4.0\%-70.8\%), 37.4\% (4.0\%-70.8\%), 43.65\% (40.0\%-47.30\%), and $37.75 \%$ (37.1\%-38.4\%), respectively.

\section{DISCUSSION}

MMP-7 gene is implicated in cancer susceptibility and metastasis in a variety of gastrointestinal-related cancers ${ }^{19}$ ${ }^{11}$. In the recent decade, several molecular epidemiological studies have been performed to assess the association of MMP-7 -181A $>$ G polymorphism with $C R C$ and GC risk. However, the results were conflicting. Thus, we conducted a comprehensive meta-analysis involving published data, to assess the strength of association of MMP-7 -181A>G polymorphism CRC and GC risk. In current meta-analysis, a total of 19 case-control studies including 3,714 cases and 4,712 controls were recruited. The subgroup analysis by cancer type, showed that there was significant association between the MMP-7 -181A>G polymorphism and increased risk of GC in overall estimations, but not with CRC. In a stratified analysis by ethnicity, our results indicated that MMP-7 $-181 A>G$ polymorphism was associated with a significantly increased risk of CRC and GC in Asians. Moreover, stratified analysis according to source of controls and genotyping method revealed a significantly increased risk of CRC and GC in participants with the MP-7 $-181 \mathrm{~A}>\mathrm{G}$ polymorphism in those studies involving PCR-RFLP, population-based and hospital-based (Table 3).

Our results were consistent with the previous metaanalyses on MMP-7 -181A $>\mathrm{G}$ polymorphism and GC risk. Thus, MMP-7 -181A>G polymorphism might serve as a susceptibility marker to GC risk. However, our results are inconsistent with the previous meta-analyses on MMP-7 $-181 \mathrm{~A}>\mathrm{G}$ polymorphism and CRC risk. In 2013, two metaanalyses by Ke et al and Yang et al were conducted to examine the association between MMP-7 -181A>G polymorphism and CRC risk ${ }^{10,31}$. Ke et al in meta-analysis of seven casecontrol have found a significant association between MMP-7 $-181 \mathrm{~A}>\mathrm{G}$ polymorphism and CRC under the homozygote model (GG vs. AA, OR=1.13, 95\% Cl=1.01-1.26) ${ }^{10}$. Similarly, Yang et al in a meta-analysis of seven studies with 1,502 CRC cases and 1,602 controls found significant association between MMP-7 -181A > G polymorphism and CRC under the homozygote model $(\mathrm{OR}=1.31,95 \% \mathrm{Cl} 1.02-1.69)^{31}$. However, their findings about MMP-7 $-181 \mathrm{~A}>\mathrm{G}$ polymorphism and CRC risk essentially remains an open field, as the number of studies is considerably smaller than that needed to yield a robust conclusion. In addition, the previous meta-analyses did not perform stratified analysis by ethnicity to identify possible association of MMP-7 -181A $>$ G polymorphism with CRC among different ethnic groups.

Between-studies heterogeneity plays an important role when performing a meta-analysis ${ }^{32}$. Heterogeneity could result from study design, genotyping error, selection bias, population stratification, sample size, allelic heterogeneity, or chance ${ }^{9,25}$. Therefore, finding the source of heterogeneity is very important for the final result of meta-analysis. Through performing sub group analysis, we found that the heterogeneity could not be explained by genotyping method, HWE status and source of controls in this meta-analysis. However, the results showed that the heterogeneity effectively removed by subgroup analysis based on ethnicity among studies on CRC, indicating that studies among Asian populations regarding $C R C$ might be a source of the heterogeneity in our meta-analysis.

This meta-analysis had three main strengths. First, this is the biggest and most recent meta-analysis of the association of MMP-7 -181A $>\mathrm{G}$ polymorphism with CRC and GC risk, and it was more powerful than previous single case-control studies. Second, this is the first meta-analysis by subgroup analysis showed that the MMP-7 -181A>G polymorphism was associated with CRC risk in Asians. Third, a comprehensive searching strategy from several electronic databases with manual search made the eligible studies included as much as possible.

Despite the clear strengths of this meta-analysis, limitations of our meta-analysis should be noted. First, although all the eligible studies were included to this metaanalysis, the sample size of the included studies was not large enough, which could increase the likelihood of type I and type II errors. Therefore, there was a lack of statistical power to better evaluate the association of MMP-7 -181A>G polymorphism with CRC and GC risk. Second, most of included studies in the present meta-analysis mainly provided data in Asians. In addition, the sample size was relatively small for stratified analyses by ethnicity and might not have provided sufficient power to estimate the association of MMP-7 -181A > G polymorphism among different ethnic groups. Third, although the funnel plot and Egger's test did not show evidence of publication bias in overall estimations, the influence of bias in the present analysis could not be completely excluded. For example, the negative findings are usually difficult to get published, or in this meta-analysis we have included only studies published in English, which produced selection bias at the start of our study. Fourth, the present meta-analysis was based primarily on unadjusted effect estimates, because most studies did not provide the adjusted OR and $95 \% \mathrm{Cl}$ controlling for potential confounding factors, thus the effect estimates were relatively imprecise. If individual data were available, adjusted ORs could be obtained to give a more precise analysis. Finally, it is well known that CRC and GC are multifactor conditions; however, the effects of gene-gene and gene-environment interactions were not addressed in the current meta-analysis.

\section{CONCLUSION}

This meta-analysis indicated that the MMP-7 -181A $>\mathrm{G}$ polymorphism might be a risk factor for susceptibility to GC in overall estimations and in Asians. However, our results clearly showed that the MMP-7 -181A>G polymorphism significantly increased the risk of CRC only in Asians. Considering the limited sample size and ethnicities, well-designed studies taking into consideration of gene-gene and gene-environment interactions should be performed to confirm our results. 
ORCID

Hossein Neamatzadeh: 0000-0003-1031-9288

\section{REFERENCES}

1. Adachi $Y$, Yamamoto $H$, Itoh F, Hinoda Y, Okada Y, Imai K. Contribution of matrilysin (MMP-7) to the metastatic pathway of human colorectal cancers. Gut. 1999 Aug 1;45(2):252-8.

2. Banday MZ, Sameer AS, Mir AH, Mokhdomi TA, Chowdri NA, Haq E. Matrix metalloproteinase (MMP) -2,-7 and -9 promoter polymorphisms in colorectal cancer in ethnic Kashmiri population - A case-control study and a mini review. Gene. 2016 Sep 1;589(1):81-9.

3. Dziki Ł, Przybyłowska K, Majsterek I, Trzciński R, Mik M, Sygut A. A/G Polymorphism of the MMP-7 Gene Promoter Region in Colorectal Cancer. Polish J Surg. 2011 Jan 1;83(11):622-6.

4. Fang W-L, Liang W-B, He H, Zhu Y, Li S-L, Gao L-B, et al. Association of Matrix Metalloproteinases 1, 7, and 9 Gene Polymorphisms with Genetic Susceptibility to Colorectal Carcinoma in a Han Chinese Population. DNA Cell Biol. 2010 Nov;29(11):657-61.

5. Fang W-L, Liang W-B, Gao L-B, Zhou B, Xiao F-L, Zhang L. Genetic polymorphisms in Matrix Metalloproteinases -1 and -7 and susceptibility to gastric cancer: an association study and meta-analysis. Iran J Allergy Asthma Immunol. 2013 Jul 9:12(3):203-10.

6. Fanjul-Fernández M, Folgueras AR, Cabrera S, López-Otín C. Matrix metalloproteinases: Evolution, gene regulation and functional analysis in mouse models. Biochim Biophys Acta - Mol Cell Res. 2010 Jan 1;1803(1):3-19.

7. Forat-Yazdi M, Gholi-Nataj M, Neamatzadeh H, Nourbakhsh P, ShakerArdakani H. Association of XRCC1 Arg399Gln polymorphism with colorectal cancer risk: A huge meta analysis of 35 studies. Asian Pacific J Cancer Prev [Internet]. 2015;16(8).

8. Ghilardi G, Biondi ML, Erario M, Guagnellini E, Scorza R. Colorectal carcinoma susceptibility and metastases are associated with matrix metalloproteinase-7 promoter polymorphisms. Clin Chem. 2003 Nov;49(11):1940-2.

9. Gohari $M$, Neámatzadeh $H$, Jafari MA, Mazaheri $M$, Zare-Shehneh $M$, Abbasi-Shavazi E. Association between the 53 codon 72 polymorphism and primary open-angle glaucoma risk: Meta-analysis based on 11 case-control studies. Indian J Ophthalmol. 2016 Oct;64(10):756-61.

10. Ke P, WuZ-D, Wen H-S, Ying M-X, Long H-C, Qing L-G. Current evidence on associations between the MMP-7 (-181A\&gt;G) polymorphism and digestive system cancer risk. Asian PacJ Cancer Prev. 2013;14(4):2269-72.

11. Kesh K, Subramanian L, Ghosh N, Gupta V, Gupta A, Bhattacharya S, et al.Association of MMP7 -181A $\rightarrow$ GPromoterPolymorphism with Gastric Cancer Risk. J Biol Chem. 2015 Jun 5;290(23):14391-406.

12. Kim JH, Pyun JA, LeeKJ, Cho SW, KwackKB. [Studyonassociation between single nucleotide polymorphisms of MMP7, MMP8, MMP9 genes and development of gastric cancer and lymph node metastasis]. Korean J Gastroenterol. 2011 Nov 25;58(5):245-51.

13. KleinT,BischoffR.Physiologyand pathophysiologyofmatrixmetalloproteases Amino Acids. 2011 Jul;41(2):271-90.

14. Kubben FJGM, Sier CFM, Meijer MJW, van den Berg M, van der Reijden JJ, Griffioen G, et al. Clinical impact of MMP and TIMP gene polymorphisms in gastric cancer. Br J Cancer. 2006 Sep 29;95(6):744-51.

15. Li J, Tian M, Zhao A. T2028 Polymorphism in the Promoter Region of the Metalloproteinase-7 Increases Susceptibility and Risk of Metastasis of Gastric Adenocarcinoma. Gastroenterology. 2008 Apr 1;134(4):A-603.

16. Lièvre A, Milet J, Carayol J, Le Corre D, Milan C, Pariente A, et al. Genetic polymorphisms of MMP1, MMP3 and MMP7gene promoter and risk of colorectal adenoma. BMC Cancer. 2006 Dec 24;6(1):270.

17. MalikMA,ZargarSA, MittalB. Roleofthemetalloproteinase-7(181A\&gt;G) polymorphism in gastric cancer susceptibility: a case control study in Kashmir valley. Asian Pac J Cancer Prev. 2011;12(1):73-6.
18. Mittal R, Patel AP, Debs LH, Nguyen D, Patel K, Grati M, et al. Intricate Functions of MatrixMetalloproteinases in Physiological and Pathological Conditions. J Cell Physiol. 2016 Dec 1;231(12):2599-621.

19. Moreno-Ortiz JM, Gutierrez-Angulo M, Partida-Pérez M, PeregrinaSandoval J, Ramírez-Ramírez R, Muñiz-Mendoza R, et al. Association of MMP7-181A/GandMMP13-77A/Gpolymorphismswithcolorectalcancer in a Mexican population. Genet Mol Res. 2014 Feb 14;13(2):3537-44.

20. Motovali-Bashi M, SadeghiZ, Hemati S. Relationship between-181(A/G) region single nucleotide polymorphisms of matrilysin gene promoter and the onset and prevalence of colorectal cancer using tetra-primer ARMS PCR and RFLP-PCR techniques [Internet]. Vol. 7, Basic \& Clinical Cancer Research. Tehran University of Medical Sciences, Cancer Institute of Iran, Cancer Research Center; 2015. 20-31.

21. Musiał K, Zwolińska D. Matrix metalloproteinases and soluble Fas/FasL system as novel regulators of apoptosis in children and young adults on chronic dialysis. Apoptosis. $2011 \mathrm{Jul} ; 16(7): 653-9$.

22. Namazi A, Forat-Yazdi M, Jafari MA, Foroughi E, Farahnak S, Nasiri R, et al.Association between polymorphisms ofERCC 5 geneand susceptibility to gastric cancer: A systematic review and meta-analysis. Asian Pacific J Cancer Prev. 2017;18(10)

23. Namazi A, Forat-Yazdi M, Jafari M, Farahnak S, Nasiri R, Foroughi E, et al. Association of interleukin-10 -1082 A/G (rs1800896) polymorphism with susceptibility to gastric cancer: meta-analysis of 6,101 cases and 8,557 controls. Arq Gastroenterol. 2018 Mar,55(1):33-40.

24. Ohtani $H$, Maeda N MY. Functional polymorphisms in the promoter regions of matrix metalloproteinase-2,-3,-7,-9 and TNF- alpha genes, and the risk of colorectal neoplasm in Japanese. Yonago Acta Med. 2009;52:47-56.

25. Sobhan MR, Mahdinezhad-Yazdi M, Aghili K, Zare-Shehneh M, Rastegar S, Sadeghizadeh-Yazdi J, et al. Association of TNF- $\alpha-308$ G \&gt; A and -238G \&gt; A polymorphisms with kneeosteoarthritisrisk:Acase-control study and meta-analysis. J Orthop. 2018 Sep 1;15(3):747-53.

26. Sugimoto $M$, Furuta $T$, Kodaira $C$, Nishino $M$, Yamade $M$, Ikuma $M$, et al. Polymorphisms of matrix metalloproteinase- 7 and chymase are associated with susceptibility to and progression of gastric cancer in Japan. J Gastroenterol. 2008 Oct 29;43(10):751-61.

27. Tak DH, Moon HS, Kang SH, Sung JK, Jeong HY. Prevalence and Risk Factors of Gastric Adenoma and Gastric Cancer in Colorectal Cancer Patients. Gastroenterol Res Pract. 2016 Dec 26;2016:1-7.

28. WooM,ParkK,NamJ,KimJC.Clinicalimplications ofmatrixmetalloproteinase-1, $-3,-7,-9,-12$, and plasminogenactivatorinhibitor-1 genepolymorphisms in colorectal cancer. J Gastroenterol Hepatol. 2007 Jul;22(7):1064-70.

29. Wu J, Guan X, Li Y-T, Bai P, Wu J. Matrix metalloproteinase7 -181A/G polymorphism is associated with increased cancer risk among highquality studies: Evidence from a meta-analysis. Clin Biochem. 2013 Nov:46(16-17):1649-54.

30. Yang T-F, Guo L, Wang Q. Meta-analysis of associations between four polymorphisms in the matrix metalloproteinases geneand gastric cancer risk. Asian Pac J Cancer Prev. 2014;15(3):1263-7.

31. Yang X, Liu Y, Yang Y, Li B. Update meta-analysis on MMP-7 -181A\&gt; G polymorphism and cancer risk: Evidence from 25 studies. Gene. 2013 Jun 1;521(2):252-8.

32. Yazdi MM, Jamalaldini MH, Sobhan MR, Jafari M, Mazaheri $M$, ZareShehneh $M$, et al. Association of ESR $\alpha$ Gene Pvu II T>C, Xbal A>G and Btgl G>A Polymorphisms with Knee Osteoarthritis Susceptibility: A Systematic Review and Meta-Analysis Based on 22 Case-Control Studies. Arch bone Jt Surg. 2017 Nov; 5(6):351-62.

33. Yueh T-C, Wu C-N, Hung Y-W, Chang W-S, Fu C-K, Pei J-S, et al. The Contribution of MMP-7 Genotypes to Colorectal Cancer Susceptibility in Taiwan. Cancer Genomics Proteomics. 2018 Apr 24;15(3):207-12.

34. Zhang J, Jin X, Fang S, Wang R, Li Y, Wang N, et al. The functional polymorphism in the matrix metalloproteinase-7 promoter increases susceptibility to esophageal squamous cell carcinoma, gastric cardiac adenocarcinoma and non-small cell lung carcinoma. Carcinogenesis. 2005 Oct 1;26(10):1748-53.

35. Zhang Y, Chen Q. Relationship between matrix metalloproteinases and the occurrence and development of ovarian cancer. Brazilian J Med Biol Res $=$ Rev Bras Pesqui medicas e Biol. 2017 May 18;50(6):e6104. 